\title{
Erratum: Mobility Measurements Probe Conformational Changes in Membrane Proteins due to Tension [Phys. Rev. Lett. 115, 198101 (2015)]
}

\author{
Richard G. Morris and Matthew S. Turner
}

(Received 11 April 2016; published 8 June 2016)

DOI: 10.1103/PhysRevLett.116.239901

Our analysis neglected the role of the Gaussian curvature $K$ in the membrane Hamiltonian, implicitly setting the saddlesplay modulus $\bar{\kappa}=0$. For the general case $\bar{\kappa} \neq 0$, Eq. (1) should contain an extra term $\bar{\kappa} K$ under the integral sign. (Note that since the shape of the protein may change, so can the geodesic curvature $c_{g}$ of the boundary between the protein and the membrane, and, as a result, the Gauss-Bonnet theorem $\int_{\mathcal{S}} K d A=2 \pi-\int_{\partial \mathcal{S}} c_{g} d l$ cannot be used to equate the additional term to a constant, as would normally be the case.) Incorporating such a saddle-splay term does not affect either the shape of the membrane [Eq. (2)] or the hydrodynamic calculations, but it does modify the expression for the torque exerted at the boundary [given by Eq. (9)] which then reads $\tau=2 \pi \alpha[a \sigma h(a)-\bar{\kappa}]$. Reflecting the data summarized in Ref. [1], we set $\bar{\kappa} \approx-0.9 \kappa$, which, with the remaining calculation and fitting procedure unchanged, leads to a modifed prediction for the torsional rigidity of $86.3 k_{B} T$, rather stiffer than originally reported. None of the discussion or our overall conclusions are otherwise affected.

[1] M. Hu, J. J. Briguglio, and M. Deserno, Biophys. J. 102, 1403 (2012). 